\begin{tabular}{|c|l|}
\hline Title & Large reduction of leakage currents in A IGaN Schottky diodes by a surface control process and its mechanism \\
\hline Author(s) & Kotani, Junji; Kaneko, Masamitsu; Hasegawa, Hideki; Hashizume, Tamotsu \\
\hline Citation & $\begin{array}{l}\text { Journal of Vacuum Science \& Technology B Microelectronics and Nanometer Structures, 24(4), 2148-2155 } \\
\text { https:/doi.org/10.1116/1.2216722 }\end{array}$ \\
\hline Issue Date & 2006-07 \\
\hline Doc URL & http://hdl.handle.net/2115/15875 \\
\hline Type & article \\
\hline File Information & JV STB24 4.pdf \\
\hline
\end{tabular}

Instructions for use 


\title{
Large reduction of leakage currents in AIGaN Schottky diodes by a surface control process and its mechanism
}

\author{
Junji Kotani, ${ }^{\text {a) }}$ Masamitsu Kaneko, Hideki Hasegawa, and Tamotsu Hashizume \\ Research Center for Integrated Quantum Electronics (RCIQE), Hokkaido University, Sapporo 060-8628, \\ Japan and Graduate School of Information Science and Technology, Hokkaido University, Sapporo \\ 060-8628, Japan
}

(Received 15 January 2006; accepted 18 March 2006; published 26 July 2006)

\begin{abstract}
Leakage currents in AlGaN Schottky diodes were investigated systematically by using a rigorous computer simulation based on the thin surface barrier model taking account of unintentionally doped surface donors. The leakage currents in AlGaN Schottky diodes have stronger bias dependence and smaller temperature dependences as compared with those of GaN diodes. It was shown that these features were associated with shallow oxygen donors located near the AlGaN surface. Then, an attempt was made to remove oxygen and suppress leakage currents by a surface control process using an ultrathin $\mathrm{Al}$ layer and subsequent annealing. An in situ x-ray photoelectron spectroscopy analysis indicated the formation of $\mathrm{Al}_{2} \mathrm{O}_{3}$ layer during the surface control process, suggesting efficient gettering of oxygen from the surface. $C-V$ analysis directly indicated the reduction of shallow donors by the surface control process. A remarkable reduction of reverse leakage currents of four to five orders of magnitude took place in large area AlGaN Schottky diodes after the application of the surface control process. This process also reduced leakage currents of the gate of the heterostructure field effect transistor device by more than one order of magnitude and increased temperature dependences of current. (C) 2006 American Vacuum Society. [DOI: 10.1116/1.2216722]
\end{abstract}

\section{INTRODUCTION}

Ever since nitride-based semiconductor materials have started to attract intense attention for optical and high-power device applications such as blue laser diodes, blue/UV light emitting diodes, and high power $\mathrm{AlGaN} / \mathrm{GaN}$ heterostructure field effect transistors (HFETs), material growth and device processing technologies have made dramatic advances. However, the state of the art of these technologies is still not quite as satisfactory as those for $\mathrm{Si}, \mathrm{GaAs}$, and InP.

One of the unsolved key issues concerning crystal growth is related to native defects and impurities. The incorporation of native crystalline defects such as gallium vacancy, ${ }^{1,2}\left(V_{\mathrm{Ga}}\right)$ and nitrogen vacancy, ${ }^{3,4}\left(V_{\mathrm{N}}\right)$ and impurities such as carbon, hydrogen, and oxygen into the crystal is not well controlled yet $;{ }^{5,6}$ neither their incorporation mechanisms nor their roles in the crystal are well understood as compared to cases of $\mathrm{Si}$, $\mathrm{GaAs}$, and InP which have been studied for a much longer time. The $V_{\mathrm{Ga}}$ defect has been found to serve as a deep acceptor related to the so-called yellow luminescence in GaN. ${ }^{1,7}$ On the other hand, the $V_{\mathrm{N}}$ defect is another well known defect in $\mathrm{GaN}$ and $\mathrm{AlGaN}$ crystals, and for quite some time, researchers have thought that it is a shallow donor as supported by a theoretical calculation, ${ }^{8}$ and that it gives rise to $n$-type background doping in $\mathrm{GaN}$ and $\mathrm{AlGaN}$. However, another theoretical calculation indicated that it is a deep donor, ${ }^{9}$ and our recent experimental study on Schottky barriers and ungated HFET structures supported the deep level nature of $V_{\mathrm{N}}{ }^{10}$

\footnotetext{
${ }^{a}$ Author to whom correspondence should be addressed; electronic mail: kotani@rciqe.hokudai.ac.jp
}

For oxygen, a recent series of analyses using techniques such as the secondary ion mass spectroscopy (SIMS) have indicated that there exist high-density oxygen atoms in GaN and $\mathrm{AlGaN} .{ }^{6,11}$ As for its role, there was a theory ${ }^{12}$ which predicted that it might act like a deep donor with large lattice relaxation similar to EL2 in GaAs. However, more recent experimental and theoretical investigations on the role of oxygen impurity in $\mathrm{GaN}$ and $\mathrm{AlGaN}$ have indicated that it is a shallow donor with an activation energy of about $30 \mathrm{meV} .{ }^{11,13}$ Furthermore, it has been suggested that the oxygen donor is the residual donor responsible for the $n$-type conduction of undoped $\mathrm{GaN}$ and $\mathrm{AlGaN} .^{14,15}$

On the other hand, a key issue related to device processing is the understanding and control of the surfaces and interfaces of GaN and AlGaN. Particularly, Schottky diodes formed on $\mathrm{GaN}$ and $\mathrm{AlGaN}$ suffer from large reverse leakage currents that are many orders of magnitude lager than the prediction of the thermionic emission (TE) model. ${ }^{16-19}$ There is also an indication that this leakage current is somehow related to the important device instability problem in HFETs called current collapse ${ }^{10}$ and also to the saturation of cutoff frequency of HFETs with respect to gate length reduction. ${ }^{20}$

In an attempt to explain the mechanism of large leakage current in GaN and AlGaN Schottky barriers, our group has proposed a thin surface barrier (TSB) model where unintentionally doped surface donors such as $V_{\mathrm{N}}$ donors reduce the Schottky barrier width and provide tunneling leakage path through the thermionic field emission transport mechanism. ${ }^{17,21}$ A computer simulation using the TSB model and assuming the presence of $V_{\mathrm{N}}$ deep donors with exponentially decaying spatial distributions has provided a fairly good overall picture for current leakage mechanisms in $\mathrm{GaN}$ 


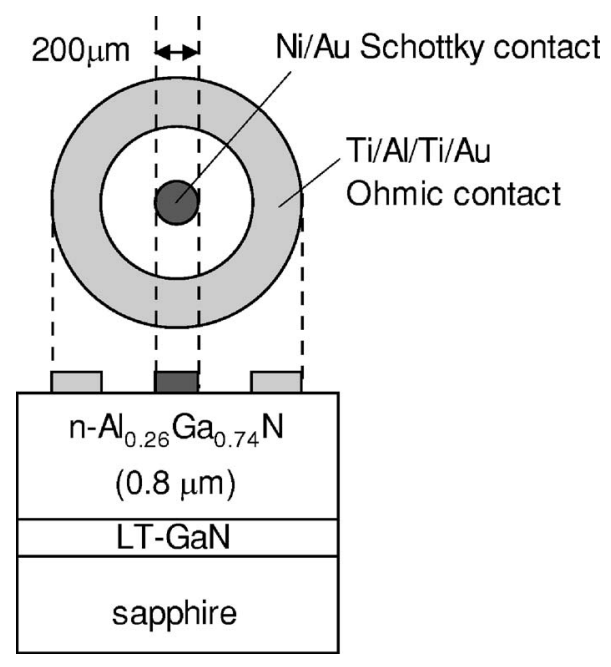

FIG. 1. Schematic illustration of a large area Schottky diode formed on a thick AlGaN layer.

and AlGaN Schottky barriers. However, as for quantitative agreement between theory and experiment, agreement has been much poorer in AlGaN Schottky barriers as compared with GaN Schottky barriers, indicating that something is still missing in the simulation framework.

The purpose of this article is to further improve the understanding of leakage currents in AlGaN Schottky interfaces by considering the presence of oxygen donors and to attempt to reduce leakage currents by applying a surface control process derived from the improved understanding of the leakage mechanism.

\section{EXPERIMENTAL AND THEORETICAL METHODS}

\section{A. Sample structure and fabrication for experimental study}

In order to investigate the current leakage behavior in the AlGaN Schottky diode in detail experimentally, large area Schottky diodes shown in Fig. 1 were prepared, and their current-voltage characteristics were measured at various temperatures. The diode had a circular Schottky electrode with a diameter of $200 \mu \mathrm{m}$ and was fabricated on a $0.8 \mu \mathrm{m}$ thick high-quality Si-doped $n$ - $\mathrm{Al}_{0.26} \mathrm{Ga}_{0.74} \mathrm{~N}$ layer which was grown on a sapphire substrate by metal organic vapor phase epitaxy (MOVPE). Typical values of the electron concentration and mobility of the Si-doped AlGaN layer at room temperature (RT) were $2 \times 10^{17} \mathrm{~cm}^{-3}$ and $100 \mathrm{~cm}^{2} / \mathrm{Vs}$, respectively. Prior to sample fabrication process, the sample surface was cleaned in organic solvents at RT and then subjected to a wet chemical treatment in an $\mathrm{NH}_{4} \mathrm{OH}$ solution at $50{ }^{\circ} \mathrm{C}$ for 5-10 min. The treatment in an $\mathrm{NH}_{4} \mathrm{OH}$ solution is effective in reducing natural oxides on AlGaN surfaces. ${ }^{22}$ As a ringshaped Ohmic contact, a $\mathrm{Ti} / \mathrm{Al} / \mathrm{Ti} / \mathrm{Au}$ layer structure was deposited on the AlGaN surface and was followed by annealing at $800{ }^{\circ} \mathrm{C}$ for $1 \mathrm{~min}$ in $\mathrm{N}_{2}$ ambient. A circular $\mathrm{Ni} / \mathrm{Au}$ Schottky electrode was formed at the center of the Ohmic ring by electron-beam deposition.

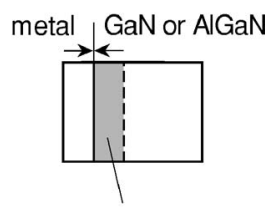

thin surface barrier (TSB) region (high-density unintentionally introduced donors)

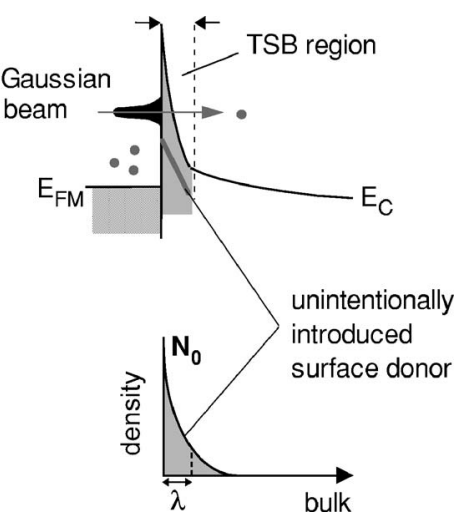

FIG. 2. Thin surface barrier (TSB) model for leakage currents.

\section{B. Method for theoretical analysis of current transport}

To analyze the current transport in $\mathrm{GaN}$ and $\mathrm{AlGaN}$ Schottky interfaces theoretically, we used, in this study, a one-dimensional (1D) rigorous simulation program which we previously developed for current transport based on the TSB model. ${ }^{17,21}$ The basic idea of the TSB model is schematically shown in Fig. 2. This model assumes that there exist unintentional donors near the Schottky interface, and this reduces the width of the Schottky barrier in such a way that a Gaussian beam of electrons can tunnel through this thin barrier in both forward and reverse directions through the thermionic field emission (TFE) transport process.The program allows the numerical calculation of TFE currents precisely for arbitrary spatial distributions of unintentional and intentional donors.

The theoretical calculation procedure was the following. The potential profile of Schottky interfaces is first calculated by solving Poisson's equation self-consistently for an arbitrary spatial distribution of a particular donor with an arbitrary energy depth $E_{\mathrm{DD}}$. Then the tunneling probability through this barrier is calculated under the Wentzel-KramersBrillouin (WKB) approximation. Finally, the current through the Schottky interface is calculated by using a general expression for the tunneling current. The details for calculation have been presented elsewhere. ${ }^{21}$

\section{ROLE OF OXYGEN IN LEAKAGE IN AIGaN SCHOTTKY DIODE}

\section{A. Problems in previous $I-V$ analysis for AIGaN Schottky diodes}

As explained in the Introduction, our computer simulation based on the TSB model gave poor quantitative agreement with experiment for AlGaN Schottky diodes as compared with GaN Schottky diodes. To explain the problems, measured and best-simulated current-voltage $(I-V-T)$ curves for $\mathrm{Ni} / \mathrm{GaN}$ and $\mathrm{Ni} / \mathrm{Al}_{0.26} \mathrm{G}_{0.74} \mathrm{~N}$ Schottky diodes are shown in Fig. 3(a) and 3(b), respectively, for measurement temperatures of 150 and $300 \mathrm{~K}$. In these analyses, the presence of a single $V_{\mathrm{N}}$-related deep donor was assumed. As for its energy depth, $E_{\mathrm{DD}}=0.25$ and $0.37 \mathrm{eV}$ were used for $\mathrm{GaN}$ and $\mathrm{Al}-$ 

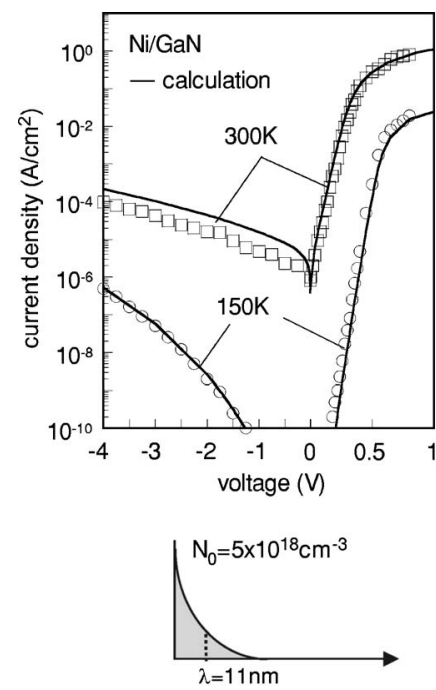

(a)
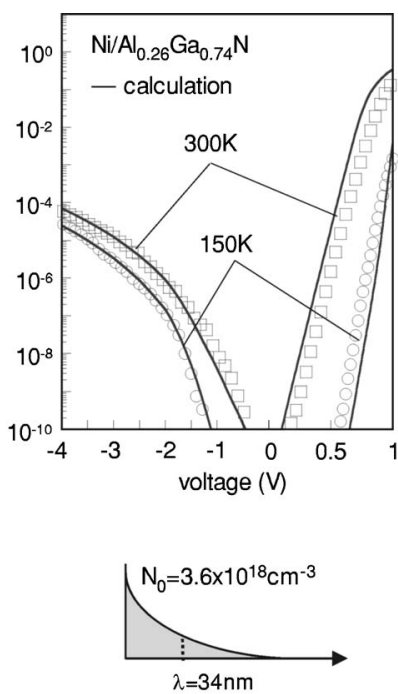

(b)

Fig. 3. (a) Measured and calculated $I-V$ - $T$ characteristics of Schottky diodes fabricated on a thick $\mathrm{GaN}$ and $\mathrm{Al}_{0.26} \mathrm{Ga}_{0.74} \mathrm{~N}$ epitaxial layer. (b) The distributions for unintentional surface donors used for simulation of $\mathrm{GaN}$ and $\mathrm{Al}_{0.26} \mathrm{Ga}_{0.74} \mathrm{~N}$ Schottky diodes.

$\mathrm{GaN}$, respectively. The value of $E_{\mathrm{DD}}=0.25 \mathrm{eV}$ was obtained in our previous study as the best value for $V_{\mathrm{N}}$ in $\mathrm{GaN}$ in $I$ - $V$ simulation. ${ }^{21}$ On the other hand, $E_{\mathrm{DD}}=0.37 \mathrm{eV}$ for $\mathrm{AlGaN}$ was directly obtained experimentally from an Arrhenius plot of recovery time constant of the drain-current transient of gateless HFETs in our previous study. ${ }^{10}$ The spatial distributions of the $V_{\mathrm{N}}$ donor which gave the best fitting result are shown at the bottom of Figs. 3(a) and 3(b). They are exponentially decaying distributions from the surface with the peak density at the surface of $N_{0}$ and the decay length of $\lambda$. Such a distribution was the best distribution in reproducing experimental $I-V$ curves, as discussed in detail previously. ${ }^{21}$ The Schottky barrier height (SBH) used for simulation were 0.85 and $1.3 \mathrm{eV}$ for $\mathrm{GaN}$ and $\mathrm{AlGaN}$ Schottky diodes, respectively.

A brief comparison of Figs. 3(a) and 3(b) may lead to an impression that agreement between experiment and theory is reasonably good for both cases. However, it is noted that the rectifying behavior of the AlGaN Schottky diode is much poorer than that of the GaN Schottky diode, showing sharply increasing and less temperature-dependent reverse characteristics. It should be noted that the reason for smaller forward currents in the AlGaN diode is due to higher Schottky barrier height. In spite of this, the $\mathrm{AlGaN}$ diode show less temperature-dependent large reverse leakage currents. In order to reproduce this behavior with an exponentially decaying distribution of $V_{\mathrm{N}}$ deep donor, we had to assume a larger amount of $V_{\mathrm{N}}$ donor for the AlGaN diode. In fact, the sheet density of $V_{\mathrm{N}}$ donor for the AlGaN diode was roughly twice as large as that of the GaN diode, if one integrates the distributions shown at the bottom of Figs. 3(a) and 3(b). We saw this type of result in all the AlGaN diodes we measured and

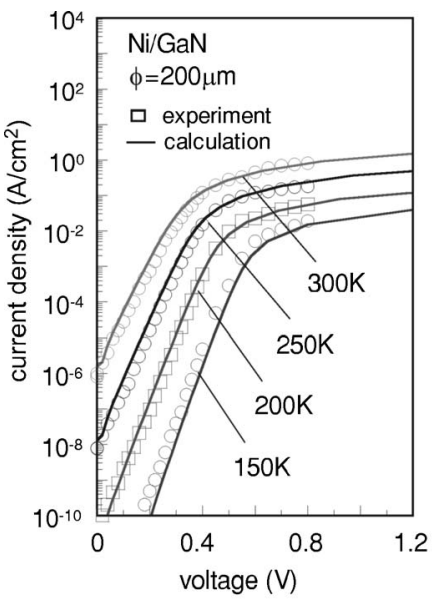

(a)

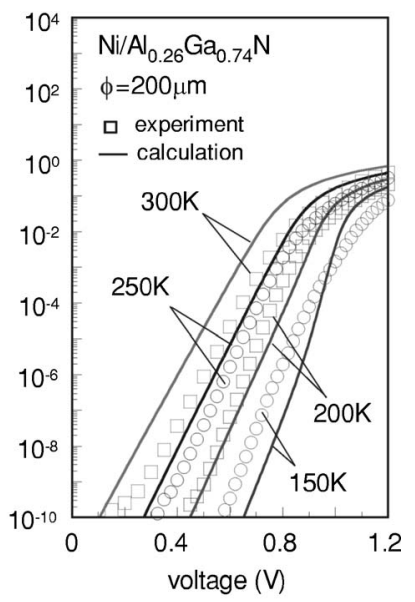

(b)
FIG. 4. Fitting results for forward bias regions of (a) Ni/GaN and (b) $\mathrm{Ni} / \mathrm{Al}_{0.26} \mathrm{Ga}_{0.74} \mathrm{~N}$ Schottky diodes using the TSB model assuming the presence of nitrogen-vacancy defects.

simulated. However, why larger numbers of $V_{\mathrm{N}}$ donors exists in $\mathrm{AlGaN}$ layers as compared with $\mathrm{GaN}$ layers was not clear at all.

Difficulty in fitting became more evident when we tried to reproduce the temperature dependences of $I-V$ curves in more detail. For example, the detailed comparison of forward $I-V$ curves obtained at temperatures of 150, 200, 250, and $300 \mathrm{~K}$ are given in Figs. 4(a) and 4(b) in an expanded scale for the same GaN and AlGaN Schottky diodes shown in Figs. 3(a) and 3(b). As seen in Fig. 4(a), the experimental results could be very well reproduced in the case of the GaN Schottky diode. On the other hand, agreement was unacceptably poor in the case of the AlGaN Schottky diode in spite of favorable overview given in Figs. 3(a) and 3(b).

In order to further understand the reason for poor fitting of the forward currents of the AlGaN diode, we carried out parameter sensitivity analysis. The calculated dependences of the forward current on $N_{0}, \lambda$, and $E_{\mathrm{DD}}$ are shown in Figs. 5(a)-5(c), respectively. We see here that the forward current do not change so much with the changes of $N_{0}$ and $\lambda$, but the change of $E_{\mathrm{DD}}$ can cause a large change in the forward current. A similar analysis for the reverse current has shown that the reverse current is not sensitive to the change in $E_{\mathrm{DD}}$, but it is very sensitive to the changes in $N_{0}$ and $\lambda$. Thus, we have to use a smaller value of $E_{\mathrm{DD}}$ or assume shallower donors to improve the fitting in spite of the fact that we directly detected the presence of $0.37 \mathrm{eV}$ deep donors in our previous study on the drain-current transient of gateless HFETs. ${ }^{10}$

\section{B. $I-V$ analysis including oxygen shallow donors}

As mentioned in the Introduction, the presence of oxygen in AlGaAs layers is a well established fact by now, and recent study has indicated that it acts as a shallow donor. The combination of this feature with the above result of the sen- 


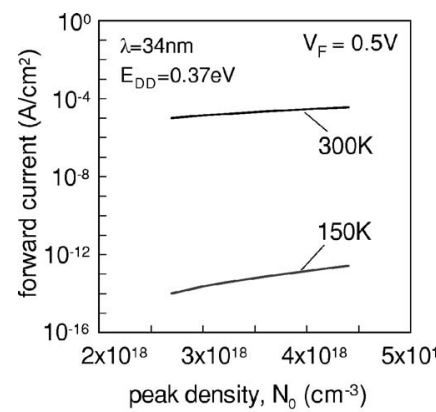

(a)

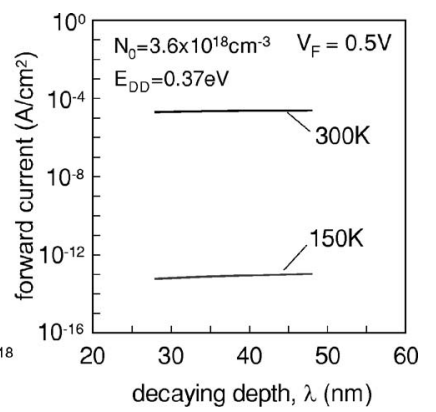

(b)

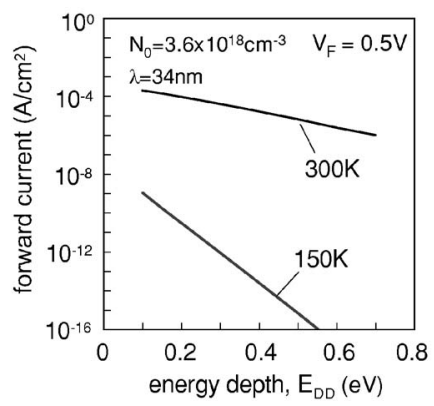

(c)

FIG. 5. Parameter sensitivity analysis for the leakage current at $V_{G}=0.5 \mathrm{~V}$ in the forward bias region vs the changes of (a) the peak density of unintentional donors $N_{0}$, (b) the decaying depth $\lambda$, and (c) the energy depth $E_{\mathrm{DD}}$, respectively. The $\mathrm{SBH}$ value of $1.3 \mathrm{eV}$ was used in the calculation.

sitivity analysis indicated to us the presence of two types of surface donors in the AlGaN layer, namely, $V_{\mathrm{N}}$ deep donor and oxygen shallow donor.

Thus, the fitting for AlGaN Schottky diodes was carried out by using two exponentially decaying distributions of these two unintentional surface donors. The energy depth of oxygen donor was assumed to be $E_{\mathrm{DD}}=0.03 \mathrm{eV}$ as previously reported by other groups. ${ }^{11,13}$

The best fitting results by using two types of unintentional donors are shown in Fig. 6(a). The assumed donor distribution and energy depths of donors are shown in Figs. 6(b) and 6(c), respectively. Compared to the result shown in Fig. 4(b), the fitting result was improved dramatically in the forward bias region, and the experimental results were reproduced very well in both forward and reverse bias regions including temperature dependence. Of course, the present analysis does not directly show that the assumed shallow donor is due to oxygen impurity. However, it can be said that is a most likely candidate. Thus, in the case of AlGaN Schottky interfaces, it is highly likely that the current transport depends on the shallow oxygen donors in addition to the deep $V_{\mathrm{N}}$ donor.

\section{REDUCTION OF LEAKAGE CURRENTS BY SURFACE CONTROL}

\section{A. Surface control process using ultrathin Al layer}

As discussed in the previous section, oxygen is likely to play a very important role in AlGaN Schottky diodes. To realize the high-quality Schottky diodes or Schottky gates in HFETs with low reverse leakage currents, the elimination of

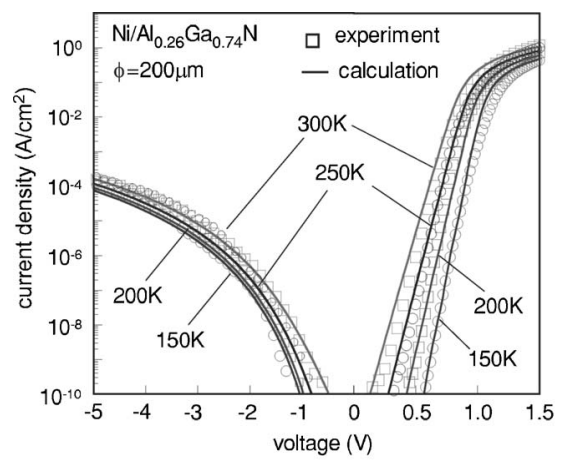

(a)

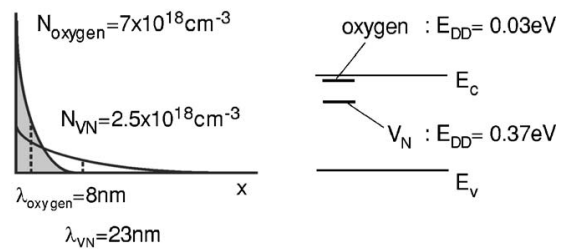

(b)

(c)

FIG. 6. (a) Fitting results assuming the presence of both nitrogen-vacancy and oxygen donors for $\mathrm{Ni} / \mathrm{Al}_{0.26} \mathrm{Ga}_{0.74} \mathrm{~N}$ Schottky diodes $(\mathrm{SBH}=1.45 \mathrm{eV})$. (b) Distribution of unintentional surface donors that gave the best fitting. The energy depths determined were 0.37 and $0.03 \mathrm{eV}$ for nitrogen-vacancy and oxygen impurities, respectively.

oxygen is thus highly desirable. Based on this idea, we attempted to develop a surface control process using ultrathin Al layer which hopefully acts as a gettering material for oxygen.

The sequence of the proposed surface control process is shown in Fig. 7. It consists of the following steps:

(1) Treatment of AlGaN surface in rf-excited plasma assisted nitrogen radicals at $300{ }^{\circ} \mathrm{C}$ for $10 \mathrm{~min}$.

(2) Molecular beam deposition of an ultrathin Al layer of $1.5 \mathrm{~nm}$ at $\mathrm{RT}$ in the molecular beam epitaxy (MBE) chamber with very slow deposition rate of $\sim 0.01 \mathrm{~nm} / \mathrm{s}$.

(3) Annealing of the sample at $700{ }^{\circ} \mathrm{C}$ for $10 \mathrm{~min}$ in ultrahigh vacuum (UHV).

(4) Removal of thin Al layer in HF solution.

(5) Electron-beam deposition of Ni/Au Schottky electrode.

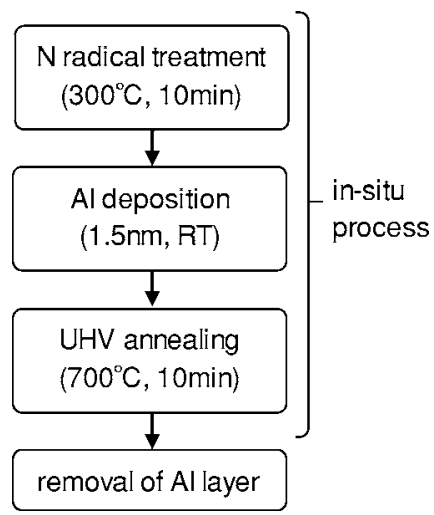

FIG. 7. Sequence of the surface control process. 


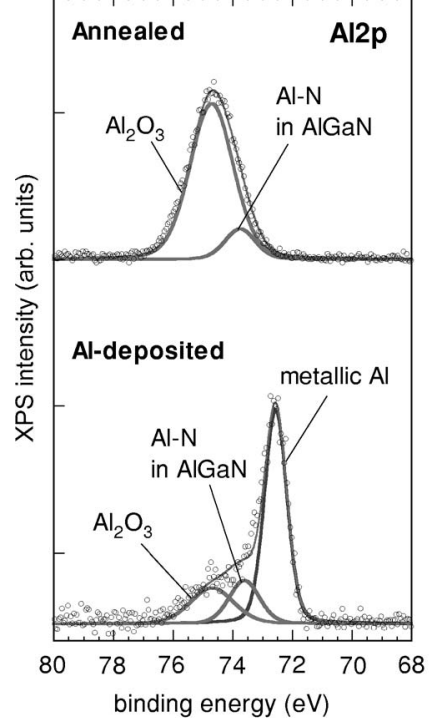

(a)

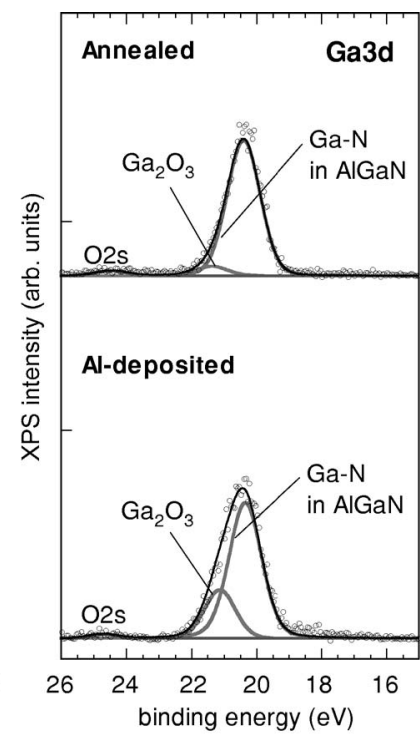

(b)
FIG. 8. In situ XPS spectra obtained from the AlGaN surface after the Al deposition and after annealing under UHV condition.

Steps (1)-(3) were carried out in situ in a UHV multichamber system without breaking the UHV condition. Step (1) was intended to reduce the $\mathrm{N}$ vacancy-related defect ${ }^{23}$ as much as possible. The most important step in the above process sequence is step (3) where out-diffusing oxygen atoms are gettered by an $\mathrm{Al} \mathrm{film.} \mathrm{On} \mathrm{the} \mathrm{other} \mathrm{hand,} \mathrm{the} \mathrm{subsequent}$ removal of the $\mathrm{Al}$ film, or an $\mathrm{Al}$ oxide film as explained later, is done outside the UHV chamber, and one may worry about the reoxidation of the surface. Our idea concerning this point is the following. The reoxidation of surface will certainly take place in air. However, no in diffusion of oxygen from the surface is expected, since step (5) is not a high temperature process like the growth of AlGaN film where oxygen atoms are incorporated.

\section{B. In situ XPS characterization of surface control process}

To clarify the surface reaction that takes place during surface control process and the possible effects of surface control process in detail, the chemical analysis was carried out by using x-ray photoelectron spectroscopy (XPS). The XPS chamber was connected with the MBE chamber by a UHV transfer chamber. Thus, after the AlGaN surface was treated in MBE chamber, it could be characterized by XPS without breaking the UHV condition.

The XPS Al $2 p$ spectra taken after Al deposition and after annealing in surface control process are compared in Fig. 8(a). Three different peaks corresponding to $\mathrm{Al}_{2} \mathrm{O}_{3}, \mathrm{AlGaN}$, and metallic $\mathrm{Al}$ were observed in the $\mathrm{Al} 2 p$ spectrum. Even immediately after the deposition of $\mathrm{Al}$ metal in UHV condition, we observed $\mathrm{Al}_{2} \mathrm{O}_{3}$ peak. This result indicates that a chemical reaction takes place immediately even at RT between the deposited Al metal and oxygen atoms existing on the $\mathrm{AlGaN}$ surface as natural oxide. As seen from Fig. 8(a), the metallic $\mathrm{Al}$ peak observed in the $\mathrm{Al} 2 p$ spectrum after $\mathrm{Al}$ deposition disappears completely by the subsequent annealing. This means that the deposited thin $\mathrm{Al}$ metal layer was oxidized completely during the annealing in UHV. According to the previous discussion, it is highly likely that highdensity oxygen impurities exist in the $\mathrm{AlGaN}$ layer. Thus, it is expected that the $\mathrm{Al}$ metal covering the $\mathrm{AlGaN}$ surface carry out the gettering action of oxygen atoms from the Al$\mathrm{GaN}$ layer, since oxygen diffusing out from the $\mathrm{AlGaAs}$ layer will quickly turn into $\mathrm{Al}_{2} \mathrm{O}_{3}$ during annealing due to the high oxygen affinity of Al. However, if we estimate the number of oxygen atoms required to form the observed $\mathrm{Al}_{2} \mathrm{O}_{3}$ layer, it is obviously much larger than that of oxygen atoms incorporated in the $\mathrm{AlGaN}$ layer. The sheet density of $\mathrm{Al}$ atoms in the $1.5 \mathrm{~nm}$ thick Al metal film is estimated to be 9.0 $\times 10^{15} \mathrm{~cm}^{-2}$ from the density of Al metal film. Thus, the number of oxygen atoms required to form an $\mathrm{Al}_{2} \mathrm{O}_{3}$ layer without any residual $\mathrm{Al}$ metal is about $1.4 \times 10^{16} \mathrm{~cm}^{-2}$. On the other hand, if the AlGaN layer includes the high-density oxygen of $1 \times 10^{18} \mathrm{~cm}^{-3}$ uniformly down to a depth of $100 \mathrm{~nm}$, the sheet density of oxygen is estimated to be only $1 \times 10^{13} \mathrm{~cm}^{-3}$. Thus, the oxygen atoms which formed the observed $\mathrm{Al}_{2} \mathrm{O}_{3}$ layer must have come from somewhere else. Thus, the above experimental result does not prove the "gettering hypothesis," although it does not disprove it.

Figure 8(b) compares the XPS Ga $3 d$ spectra taken after $\mathrm{Al}$ deposition and after annealing in the surface control process. Here, the reduction of $\mathrm{Ga}_{2} \mathrm{O}_{3}$ peak was observed after annealing as seen in the top of Fig. 8(b), although $\mathrm{Al} 2 p$ spectrum showed the oxidization of the $\mathrm{Al}$ metal. These clear changes of $\mathrm{Al} 2 p$ and Ga $3 d$ spectra indicate that the oxidation due to the residual oxygen gas in the UHV chamber is negligible and that $\mathrm{Ga}_{2} \mathrm{O}_{3}$ was decomposed during annealing, supplying oxygen atoms for the formation of $\mathrm{Al}_{2} \mathrm{O}_{3}$. This again indicates the high oxygen affinity of Al. Thus, although it is difficult to directly prove, it seems highly likely that oxygen atoms in the AlGaN layer will diffuse toward surface and will participate in the oxidation of $\mathrm{Al}$ atoms.

\section{C. $C-V$ characterization of surface control process}

A more sensitive detection technique for shallow donors is obviously the capacitance-voltage $(C-V)$ technique. To investigate the change of shallow donor distribution profiles caused by the surface control process, Schottky barriers were formed on the AlGaN surface before the surface control process and on those after the surface control with the UHV annealing times of 5 and $10 \mathrm{~min}$, and they were investigated by the $C-V$ technique. Figure 9(a) shows the resultant $1 / C^{2}$ vs $V$ plot. The measurement was carried out at $100 \mathrm{kHz}$ at $300 \mathrm{~K}$. It should be noted that the measurement was limited in the bias range of $\omega C \ll$ conductance $G$ to keep the reliability of measurement. As shown in Fig. 9(a), the plot showed slope changes in the large bias region together with additional delicate slope changes near zero bias region. The linear extrapolation of the plots near the zero bias region gave 


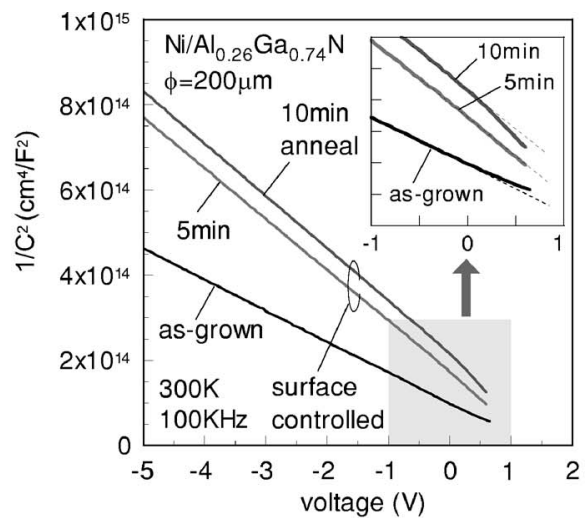

(a)

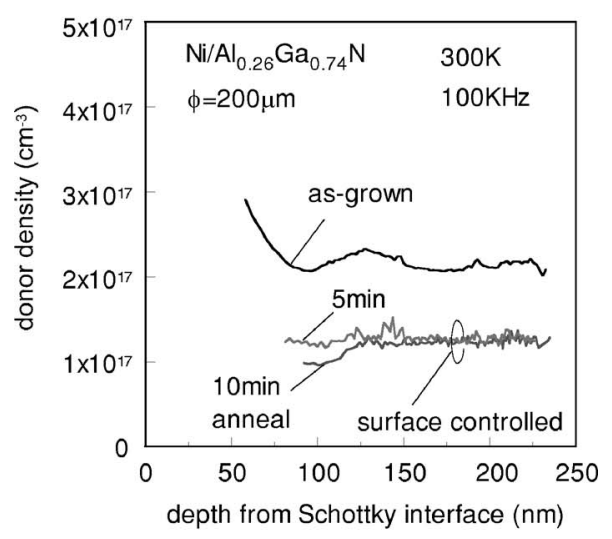

(b)

FIG. 9. (a) $1 / C^{2}-V$ plots and (b) donor concentration profiles of the AlGaN Schottky diodes without and with the surface control process.

nearly the Schottky barrier height of about $1.4 \mathrm{eV}$, confirming the formation of similar Schottky barriers for the three samples.

Furthermore, the donor profiles in depth direction were obtained from results of $C-V$ measurements, and the result is shown in Fig. 9(b). The clear difference was seen between the AlGaN Schottky diodes before and after the surface control process. A clear increase of donor concentration toward surface was observed near the surface region of $\sim 100 \mathrm{~nm}$ before the surface control process. After the surface control, this near-surface increase disappeared in two samples. Additionally, although the donor concentration before the surface control process showed a constant bulk donor density of $N_{D}=2 \times 10^{17} \mathrm{~cm}^{-3}$ in the relatively deep region over $100 \mathrm{~nm}$, it was reduced down to constant $N_{D}$ of $1.2 \times 10^{17}$ and $1.1 \times 10^{17} \mathrm{~cm}^{-3}$ for the samples with annealing of 5 and 10 min, respectively as seen in Fig. 9(b). On the other hand, the Si doping density of the wafer was $1.1 \times 10^{17} \mathrm{~cm}^{-3}$. Furthermore, it has been reported that Si donors have excellent thermal stability in nitrides and it does not redistribute by annealing even at $800{ }^{\circ} \mathrm{C} .{ }^{24}$ On the other hand, the diffusion of oxygen atoms by annealing at $500-900{ }^{\circ} \mathrm{C}$ has been reported for $\mathrm{SiO}_{2} / \mathrm{GaN}$ interfaces. ${ }^{25}$ Thus, it is highly likely that oxygen had a decaying distribution in the AlGaN layer before the surface control process and that this distribution

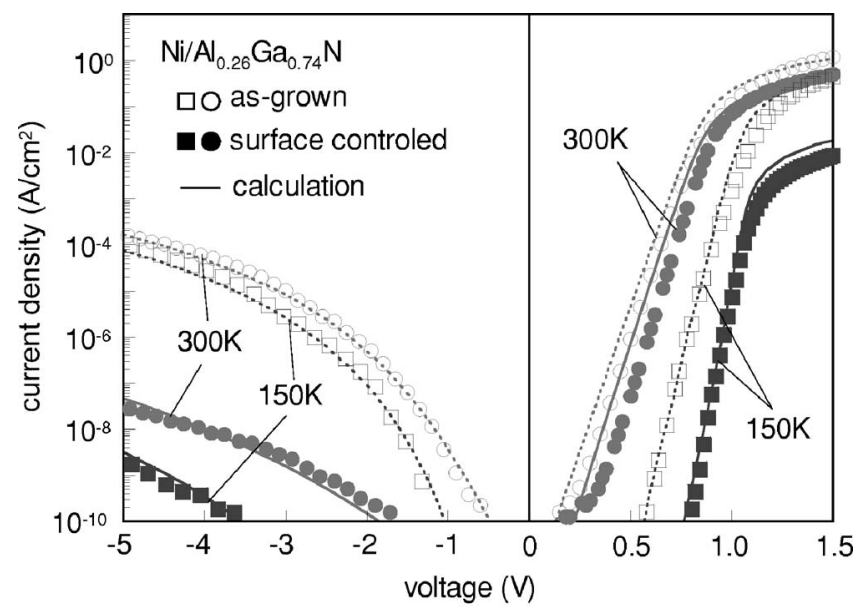

(a) as-grown

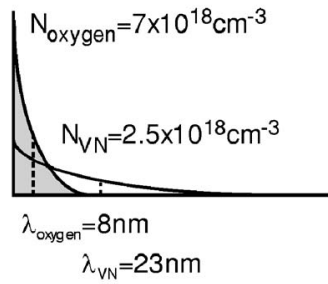

(b) surface controlled

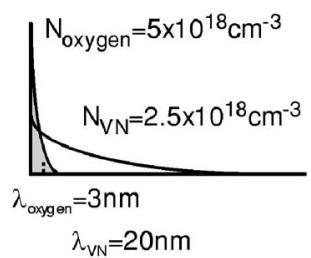

(c)
FIG. 10. (a) $I-V-T$ characteristics of the $\mathrm{Ni} / \mathrm{Al}_{0.26} \mathrm{Ga}_{0.74} \mathrm{~N}$ Schottky diodes without and with the surface control process. (b) Distribution of surface donors determined by fitting.

was changed during the surface control process at $700{ }^{\circ} \mathrm{C}$. The result indicates that the present surface control process efficiently removes oxygen donors having a decaying distribution from the surface and realizes the intrinsic constant donor density of Si.

\section{Effect of surface control process on $\mathrm{I}-\mathrm{V}$ characteristics and its simulation}

Figure 10(a) compares the $I-V$ characteristics of $\mathrm{Ni} / \mathrm{AlGaN}$ Schottky diodes fabricated with and without the surface control process, respectively. As mentioned in the previous section, the Schottky diode fabricated without the surface control process exhibited large reverse leakage currents. On the other hand, a remarkable reduction of reverse leakage current of four to five orders of magnitude took place by applying the proposed surface control process aimed at the elimination of oxygen donors. Furthermore, the surface control process led to the increase of temperature dependences of both forward and reverse currents, as seen in Fig. 10(a).

The best fitting results based on the TSB model taking account of two types of surface donors are also shown in Fig. 10(a) by solid lines. Here, the exponentially distributed shallow donors and deep donors shown in Fig. 10(b) were assumed for calculation. $N_{\text {oxygen }}=7 \times 10^{18} \mathrm{~cm}^{-3}, \lambda_{\text {oxygen }}=8 \mathrm{~nm}$, $N_{V_{\mathrm{N}}}=2.5 \times 10^{18} \mathrm{~cm}^{-3}$, and $\lambda_{V_{\mathrm{N}}}=23 \mathrm{~nm}$ were used to obtain 
the best fitting result for the diode before the surface control process. In terms of the sheet concentration of defects, these correspond to $N s_{\text {oxygen }}=5.6 \times 10^{12} \mathrm{~cm}^{-2}$ and $N s_{V_{\mathrm{N}}}=5.75$ $\times 10^{12} \mathrm{~cm}^{-2}$, respectively. For the diode after the surface control process, $N_{\text {oxygen }}=5.0 \times 10^{18} \mathrm{~cm}^{-3}$, and $\lambda_{\text {oxygen }}=3 \mathrm{~nm}$, $N_{V_{\mathrm{N}}}=2.5 \times 10^{18} \mathrm{~cm}^{-3}$, and $\lambda_{V_{\mathrm{N}}}=20 \mathrm{~nm}$ gave the best fitting results which correspond to sheet concentrations of $N s_{\text {oxygen }}$ $=1.5 \times 10^{12} \mathrm{~cm}^{-2}$ and $N s_{V_{\mathrm{N}}}=5.0 \times 10^{12} \mathrm{~cm}^{-2}$. In accordance with the characterization results using $C$ - $V$ curves, 1.1 $\times 10^{17} \mathrm{~cm}^{-3}$ was used for the surface controlled AlGaN Schottky diodes as the bulk uniform donor density.

Theoretical calculation reproduced experimental $I-V$ curves remarkably well for different temperatures, and this indicated the large reduction of oxygen impurities by the surface control process. The observed large reduction of the leakage current and increase of temperature dependences of the reverse and forward currents can be attributed to the reduction of oxygen donors by the surface control process as indicated by simulation.

However, one point which deserves further consideration is the behavior of the $V_{\mathrm{N}}$ defect. Since the oxygen atom occupies the nitrogen site so as to serve as a substitutional shallow donor, ${ }^{11,13}$ the out diffusion of oxygen implies a corresponding increase of the density of the $V_{\mathrm{N}}$ defect. However, our result of theoretical fitting to experiments indicated that the density of the $V_{\mathrm{N}}$ defect slightly reduced after annealing.

Our explanation for this apparent discrepancy is the following. Although $V_{\mathrm{N}}$ defects are most probably formed by oxygen out diffusion, it is highly likely that resultant $V_{\mathrm{N}}$ defects also diffuse out in a manner similar to the case of out diffusion of defects during thermal annealing after the ion implantation process. Such a postimplantation annealing process is known to require a cap layer to prevent the generation of vacancies of volatile group- $\mathrm{V}$ atoms at the surface. In our case, the $\mathrm{Al}_{2} \mathrm{O}_{3}$ layer mentioned previously can serve as such a cap layer. Furthermore, the out diffusion of $V_{\mathrm{N}}$ defects may form an Al-Ga metallic layer underneath the cap layer, but such a layer will be readily oxidized to become a part of the cap layer, as indicated by the strong tendency of oxidation observed in the above XPS analysis. Thus, the $V_{\mathrm{N}}$ concentration profile remained unchanged or even decreased due to the out diffusion of $V_{\mathrm{N}}$ defects.

\section{E. Application of surface control to AIGaN/GaN HFET}

To investigate the applicability of the surface control process to the reduction of leakage currents of the nanometerscale Schottky gate of the HFET device, experiments were also done on the AlGaN/GaN HFET shown in Fig. 11(a). The device was fabricated on an $\mathrm{AlGaN} / \mathrm{GaN}$ heterostructure wafer also grown by MOVPE. Typical values of the electron concentration and mobility of the two-dimensional electron gas (2DEG) were $1.0 \times 10^{13} \mathrm{~cm}^{-3}$ and $1100 \mathrm{~cm}^{2} / \mathrm{V} \mathrm{s}$, respectively, at RT. For HFET fabrication, a mesa isolation process was carried out by using an electroncyclotron resonance assisted reactive ion beam etching using a $\mathrm{CH}_{4} / \mathrm{H}_{2} / \mathrm{Ar} / \mathrm{N}_{2}[5 / 15 / 3 / 3 \mathrm{SCCM}$ (standard cubic centi-
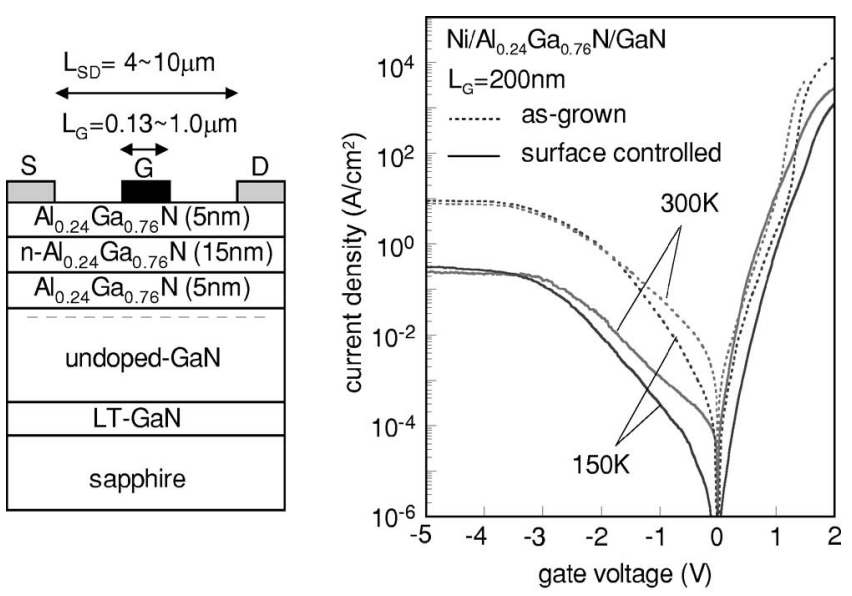

(a)

(b)

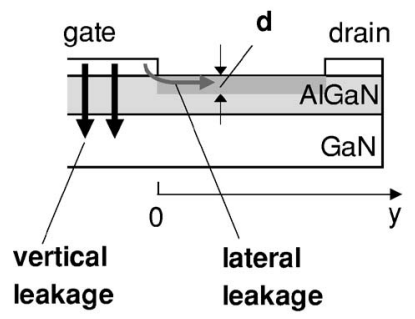

(c)

FIG. 11. (a) Schematic illustration of an AlGaN/GaN HFET sample having a nanometer-scale Schottky gate and (b) the reduction of gate leakage currents of the AlGaN/GaN HFET sample caused by the surface control process. (c) Schematic illustration of vertical and lateral tunneling current components in the gate leakage current of the $\mathrm{AlGaN} / \mathrm{GaN}$ HFET sample.

meter per minute)] mixed gas system developed by our group. ${ }^{26} \mathrm{Ti} / \mathrm{Al} / \mathrm{Ti} / \mathrm{Au}$ and $\mathrm{Ni} / \mathrm{Au}$ metal layers were used as Ohmic electrodes and Schottky electrodes, respectively. The gate length $L_{G}$ of the device was changed from 130 to $1000 \mathrm{~nm}$, while the gate width $W_{G}$ was fixed at $60 \mu \mathrm{m}$. No surface passivation was applied to all the devices fabricated in this study.

$I-V$ curves obtained on AlGaN/GaN HFETs fabricated without and with the surface control process are compared in Fig. 11(b) by solid lines. The dashed curves represent the $I-V$ curves measured on the AlGaN/GaN HFET without the surface control process, and the solid curves are those for the device with the surface control process, respectively. It is seen that, after the surface control process, the reverse leakage current was decreased with more than one order of magnitude in the whole reverse bias region, and the temperature dependence also increased in forward bias region. These improvements of gate leakage characteristics come from the reduction of oxygen impurities related to shallow donors in AlGaN layer. More precisely, the reduction of reverse leakage currents comes from the reduction of total amount of surface donors, and the improvement of temperature dependence in forward bias region results from the elimination of shallow donors, as discussed above.

It should also be noted here that the leakage current characteristics of the nanometer-scale Schottky gate in the HFET 
device is not so simple as compared to those of large area Schottky diodes. We should take account of the fact that the lateral tunneling injection occurs in gate edge as shown in Fig. 11(c). The detailed discussion about the lateral tunneling current has been published elsewhere. ${ }^{20,27}$ Briefly, if highdensity oxygen impurities exist in the surface layer, they induce concentration of electric field lines in the lateral direction along the $\mathrm{AlGaN}$ surface, and this causes large leakage currents in the low bias region due to the lateral tunneling injection of electrons. On the other hand, the vertical tunneling leakage current usually becomes dominant in high bias region. As seen in Fig. 11(b), the reduction of gate leakage currents was observed in the whole reverse bias region not only high or low bias region. Thus, the surface control process is effective in reducing both leakage current components. The mechanism of reduction of vertical tunneling currents is same as that in the large area AlGaN Schottky diodes. For lateral tunneling currents, it is likely that the high electric field in the lateral direction is relaxed by the reduction of oxygen donors near AlGaN surface.

However, the beneficial effects of the surface control process observed in nanometer-scale Schottky gate on AlGaN/GaN HFETs are unfortunately smaller than those of large area Schottky diodes formed on a thick AlGaN epitaxial layer. This is primarily due to the fact that the AlGaN barrier layer of $\mathrm{AlGaN} / \mathrm{GaN}$ wafer for HFET is Si doped to a very high density of $2 \times 10^{18} \mathrm{~cm}^{-3}$. Unfortunately, the presence of such a density of $\mathrm{Si}$ donors tend to mask the large change in the concentration of unintentionally doped oxygen donors because the $\mathrm{Si}$ donors remain in $\mathrm{AlGaN}$ layer even after surface control process by its high thermal stability. ${ }^{19}$ Furthermore, the surface portion surrounding the gate periphery of $\mathrm{AlGaN} / \mathrm{GaN}$ HFETs used in this study is exposed to air. It will introduce high densities of surface states, and the resultant pinning of the surface Fermi level can produce a strong lateral field. This may also be part of the reason for reduced beneficial effects of the surface control process. Thus, the suitable surface passivation process may be necessary for the further increase of the effects of the surface control.

\section{CONCLUSION}

In this article, we investigated the leakage currents in AlGaN Schottky interfaces by comparing experiments with computer simulations based on the TSB model involving unintentional surface donors. To reduce leakage currents of AlGaN Schottky interfaces, a surface control process was developed. The main conclusions are listed below.

(1) The leakage currents in $\mathrm{Al}_{0.26} \mathrm{Ga}_{0.74} \mathrm{~N}$ Schottky diodes can be explained by including shallow oxygen donor near surface in addition to nitrogen vacancy that is dominant in GaN. The high-density oxygen impurities play a more important role in AlGaN Schottky diodes.

(2) The characterization of surface reaction during surface control process using XPS showed the reduction of $\mathrm{Ga}_{2} \mathrm{O}_{3}$ and increase in $\mathrm{Al}_{2} \mathrm{O}_{3}$ after annealing. This result indicates that $\mathrm{Al}$ metal reacts with oxygen effectively, indicating efficient gettering of oxygen shallow donors from AlGaN layer.

(3) The $C-V$ characterization of Schottky diodes showed the increase of shallow donor density toward surface before the surface control which completely disappears by applying surface control process.

(4) The surface control process is effective in reducing the leakage current not only in large area AlGaN Schottky interfaces but also nanometer-scale Schottky gate on $\mathrm{AlGaN} / \mathrm{GaN}$ HFETs. A remarkable reduction of reverse leakage currents of four to five orders of magnitude and more than one order of magnitude took place in $\mathrm{Al}_{0.26} \mathrm{Ga}_{0.74} \mathrm{~N}$ Schottky diodes and $\mathrm{AlGaN} / \mathrm{GaN}$ HFETs, respectively. An increase of temperature dependence of currents was also observed in agreement with simulation.

\section{ACKNOWLEDGMENTS}

This work is supported in part by the 21st Century COE program of "Meme-media technology approach to the R\&D of next-generation ITs" from MEXT, Japan.

${ }^{1}$ K. Saarrinen et al., Phys. Rev. Lett. 79, 3030 (1997).

${ }^{2}$ A. Uedono et al., J. Appl. Phys. 90, 181 (2001).

${ }^{3}$ T. L. Tansley and R. J. Egan, Phys. Rev. B 45, 10942 (1992).

${ }^{4}$ P. Perlin et al., Phys. Rev. Lett. 75, 296 (1995).

${ }^{5}$ D. Meister et al., J. Appl. Phys. 88, 1811 (2000).

${ }^{6}$ J. E. Van Nostrand, J. Solomon, A. Saxler, Q.-H. Xie, D. C. Reynolds, and D. C. Look, J. Appl. Phys. 87, 8766 (2000).

${ }^{7}$ D. C. Look, Phys. Status Solidi B 228, 293 (2001).

${ }^{8}$ J. Neugebauer and C. G. Van de Walle, Phys. Rev. B 50, 8067 (1994).

${ }^{9}$ E. Yamaguchi and M. R. Junnarkar, J. Cryst. Growth 189/190, 570 (1998).

${ }^{10}$ H. Hasegawa, T. Inagaki, S. Ootomo, and T. Hashizume, J. Vac. Sci. Technol. B 21, 1844 (2003).

${ }^{11}$ K. H. Ploog, and O. Brandt, J. Vac. Sci. Technol. A 16, 1609 (1997).

${ }^{12}$ C. G. Van de Walle, Phys. Rev. B 57, 2033 (1998).

${ }^{13}$ A. J. Ptak et al., Appl. Phys. Lett. 79, 2740 (2001).

${ }^{14}$ M. Ilegems and H. C. Montgomery, J. Phys. Chem. Solids 34, 885 (1973).

${ }^{15}$ B. Monemar and O. Lagerstedt, J. Appl. Phys. 50, 6480 (1979).

${ }^{16}$ A. C. Schmitz, A. T. Ping, M. A. Kahn, Q. Chen, J. W. Yang, and I. Adesida, J. Electron. Mater. 27, 255 (1998).

${ }^{17}$ H. Hasegawa and S. Oyama, J. Vac. Sci. Technol. B 20, 1647 (2002).

${ }^{18}$ E. Monroy et al., Phys. Status Solidi A 188, 367 (2001).

${ }^{19}$ X. A. Cao, S. J. Pearton, G. Dang, A. P. Zhang, F. Ren, and J. M. Van Hove, Appl. Phys. Lett. 75, 4130 (1999).

${ }^{20}$ J. Kotani, S. Kasai, T. Hashizume, and H. Hasegawa, J. Vac. Sci. Technol. B 23, 1799 (2005).

${ }^{21}$ J. Kotani, T. Hashizume, and H. Hasegawa, J. Vac. Sci. Technol. B 22, 2179 (2004).

${ }^{22}$ T. Hashizume, S. Ootomo, R. Narasaki, S. Oyama, and M. Kihara, Appl. Phys. Lett. 76, 2880 (2000).

${ }^{23}$ T. Hashizume, S. Ootomo, S. Oyama, M. Konishi, and H. Hasegawa, J. Vac. Sci. Technol. B 19, 1675 (2001).

${ }^{24}$ R. G. Wilson, S. J. Pearton, C. R. Abernathy, and J. M. Zavada, Appl. Phys. Lett. 66, 2238 (1995).

${ }^{25}$ S. J. Pearton, H. Cho, J. R. LaRoche, F. Ren, R. G. Wilson, and J. W. Lee, Appl. Phys. Lett. 75, 2939 (1999).

${ }^{26} \mathrm{H}$. Hasegawa, T. Muranaka, S. Kasai, and T. Hashizume, Jpn. J. Appl. Phys., Part 1 42, 2375 (2003).

${ }^{27}$ J. Kotani, S. Kasai, H. Hasegawa, and T. Hashizume, e-J. Surf. Sci. Nanotechnol. 3, 433 (2005). 